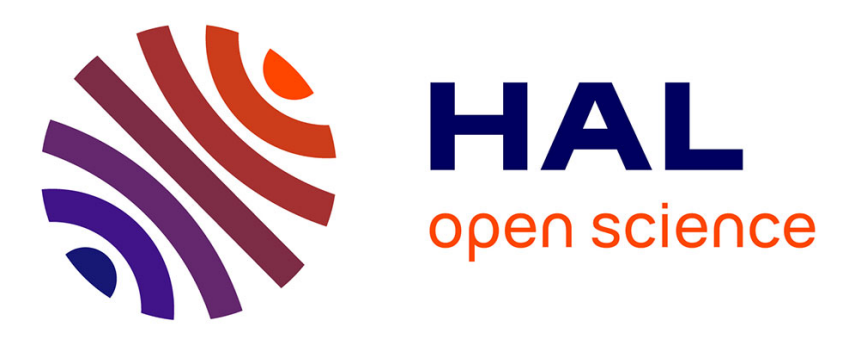

\title{
Flocking Rules Governing Swarm Robot as Tool to Describe Continuum Deformation
}

\author{
Ramiro Dell'Erba
}

\section{To cite this version:}

Ramiro Dell'Erba. Flocking Rules Governing Swarm Robot as Tool to Describe Continuum Deformation. Advanced Structured Materials, 2020, Dynamics, Strength of Materials and Durability in Multiscale Mechanics, pp.223 - 243. 10.1007/978-3-030-53755-5_14 . hal-03119201

\section{HAL Id: hal-03119201 https://hal.science/hal-03119201}

Submitted on 23 Jan 2021

HAL is a multi-disciplinary open access archive for the deposit and dissemination of scientific research documents, whether they are published or not. The documents may come from teaching and research institutions in France or abroad, or from public or private research centers.
L'archive ouverte pluridisciplinaire HAL, est destinée au dépôt et à la diffusion de documents scientifiques de niveau recherche, publiés ou non, émanant des établissements d'enseignement et de recherche français ou étrangers, des laboratoires publics ou privés. 


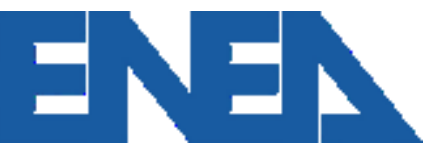

Italian National Agency for New Technologies, Energy and Sustainable Economic Development

http://www.enea.it/en http://robotica.casaccia.enea.it/index.php?lang=en

This paper is a pre-print. The final paper is available on:

dell'Erba R. (2021) "Flocking Rules Governing Swarm Robot as Tool to Describe Continuum Deformation." In: dell'Isola F., Igumnov L. (eds) Dynamics, Strength of Materials and Durability in Multiscale Mechanics. Advanced Structured Materials, vol 137. Springer, Cham. https://doi.org/10.1007/978-3-030-53755-5_14 


\title{
Flocking rules governing swarm robot as tool to describe continuum deformation
}

\author{
Ramiro dell'Erba \\ ENEA Technical Unit technologies for energy and industry - Robotics Laboratory
}

\begin{abstract}
In robotic swarm the position of an element is often determined by the behaviour of its neighbours. Following this concept we have realized a tool able to give a visually plausible simulation of continuum deformation. Without solving Newton's equation we reproduced some behaviour of bi-dimensional deformable bodies both according to the standard Cauchy model and second gradient theory. Fracture can be easily managed. The tool has computational cost advantage and it is very flexible to adapt for complex geometry samples.
\end{abstract}

\section{Keywords}

Swarm robotics, discrete mechanical systems

\section{Introduction}

Aim of this paper is not to compute physical process but to generate visually plausible simulation results with low computational cost, sacrificing some accuracy, with respect of solution of heavy differential equations, solved by finite element methods (FEM). The dynamic simulation of mechanics has its roots in computer graphics for videogames; classical methods are based on discretization (Lagrangian or Eulerian) of Newton's second law and formulate forces for each mechanical effect. Owing to the powerful of GPU some new methods become popular. The tool we propose is based on Position based dynamics (PBD) [1], widely used in computer animation due to its efficiency, robustness and simplicity. Like the robot swarm behaviour [2], [3], it do not determine forces and solve differential equations but use a position-based approach, where the new position of a particle is determined by its neighbour's positions and can be easily be used to describe complex objects. So far, using the flocking rules employed in underwater robotic swarms, to compute the displacement of the elements to achieve an assigned swarm configuration [4]-[6], we adapted the swarm control algorithm in PBD problems. Note that most of PBD methods hide the dynamics inside their relationship; moreover they ask for the knowledge of the velocity of the particles. We try to describe the deformation of a Continuum medium by this tool useful for complex micro-structures not easily analyzed by Cauchy Continuum. Classical Cauchy continua are not able to give accuracy prediction in highly non-homogeneous microstructure, though generalizations have to be introduced either considering additional degrees of freedom, to account for the kinematics at the level of the microstructure, or including in the deformation energy density higher gradients of the displacement than the first one. The latter is a particularly relevant topic if you consider the technological interest in developing exotic mechanical metamaterials able to perform targeted tasks; therefore the investigation of new and efficient algorithms is of great interest at the moment. In our tool, according to our experience in control of robot swarm, the new position of a particle is determined by the spatial position of its neighbours. The system [7]-[9] exhibits very different behaviours changing lattice type and its internal 
parameters. Therefore the compute of new position for a particle set can be considered as a constrained geometrical problem leading to a transformation operator between the matrices representing the particles configuration, $C_{t}$, for a discrete set of time steps $t 1, t 2, \ldots t_{n}$. In this paper we resume some results and try to examine the behaviour of a two dimensional beam under bending stressing what still needs to be improved.

\section{Tool description}

A complete description of the algorithm is reported in [8], [10]. Here we briefly resume it. The two dimensional continuum bodies is discretized into a finite number of particles occupying, in their initial configuration, the nodes of a lattice, chosen between the five plane Bravais lattices more honey comb lattice (Choice 1).

The lattice has four kinds of particles, but it is easy to introduce new kinds, to describe other properties, owing the modular structure of the algorithm.

1. The leaders; their motion is assigned and determine the displacement of the other particles.

2. The followers; their motion is determined by the interaction rule with other particles.

3. The frame; they are introduced so that any particle have the same number of neighbours, to avoid edge effects. Their motion is determined by the frame rule.

4. The ghost; they are introduce to describe fracture mechanism.

Choice the neighbours of any particles (Choice 2), typically the firs $n c$ particles, where $n c$ is the coordination number of the lattice (First gradient); the position of this points will determine the displacement of the point under investigation. Choice the flocking rule (Choice 3) between the particle; it describes the position of a particle as function of the neighbour's positions. Build a frame surrounding the body by an external shell of point, so that any follower interacts with the same number of elements, to avoid edge effects. Displacement of the frame point follows the motion of an assigned follower of its competence; in case the assigned followers are more (i.e. in a corner) then an average displacement, or a more generic complex rule (Choice 4), is considered. Choice a threshold, $d f$, (or a more complex rule) to be overcome to generate fracture (Choice 5). We assume that if the distance between points is larger than this threshold they stop to influence each other so they are no longer taken into account in the calculation of the follower position and fracture is declared. To balance computation of the point's displacements we introduce ghost points, to avoid collapse. Possible position, where we put ghost points (Choice 6), is that is able to recover the original shape of the lattice. Anyway other choices lead to different results and our intention will be to use different rules in order to approximate different constitutive equations. Now, by the displacements of the leaders, we can start an iterative work flow until an equilibrium state is reached. We would like underline this point in each of the possible choice; as example if we take a larger $n c$, like the neighbours of the neighbours we are in second gradient theory case. The algorithm is suitable to change particle role and rules dynamically at each time step. The most natural choice, concerning interaction rule, is the centre of gravity rule where the new $\mathrm{x}$ coordinate of the particle $\mathrm{j}$ is

$$
x_{j}(t)=\frac{\sum_{k=2}^{\text {all neighbours of } j} x_{k}(t)}{N}
$$

Possible generalizations of Eq.1 are geometric, power and weighted mean. Possible weight is the particles Euclidean distances $\operatorname{dis}(k, j)$ between the particles $k$ and $j$. This can simulate Hook law, where force is increased with increasing deformation. By Eq.1 we note as $x$ and $y$ coordinate are independent so Poisson effect cannot be obtained. A possibility is to use

$y_{j}(t)=K *\left(x_{j}(t)-x_{j}\left(t_{0}\right)\right) * d a+\frac{\sum_{k=2}^{\text {all neighbours of } j} y_{k}(t)}{N}$

Where $d a$ is a function of the distance from the central axis, $K$ a parameter determining the response force and $x\left(t_{0}\right)$ the $x$ coordinate at time $t_{0}$. So far expansion of $x$ coordinate has effect on the $y$ coordinate. The Euclidean distance, $\operatorname{dis}(k, j)$, can be used as weight.

$x_{j}(t)=\frac{\sum_{k=2}^{\text {all neighbours of } j} \operatorname{dis}(k, j) x_{k}(t)}{\sum_{k=2}^{\text {all neighbours of } j} \operatorname{dis}(k, j)}$

(3)

We can also force the follower's movement to overcome the barycentre equilibrium position, leading the lattice to oscillate. 
$x_{j}(t)=\frac{\sum_{k=2}^{\text {all neighbours of } j} w(k, j) x_{k}(t)}{\sum_{k=2}^{\text {all neighbours of } j} w(k, j)}+f d\left(\frac{\sum_{k=2}^{\text {all neighbours of } j} w(k, j) x_{k}(t)}{\sum_{k=2}^{\text {all neighbours of } j} w(k, j)}-M T\left(j, t_{0}\right)\right)$

Where $w(k, j)$ is the weight, $f d$ is a feedback factor and $M T\left(i, t_{0}\right)$ is the $x$-coordinate of $j$ point at $t_{0}$.

We are considering to discuss the proposed model in a fully variational setting, which is by no means trivial but would provide clear methodological advantages (see [11] for an introduction and [12]-[16] for illustrative cases concerning continua with non-classical properties). To this aim we introduce two formulations PE1 and PE2 concerning the pseudo-energy concept. The first is the sum, extended to the neighbours, of squares of the differences between the distances of the point from its neighbours minus the distance in the initial configuration i.e.

$$
\operatorname{PE1}(t, j)=\sum_{k=1}^{\text {all neighbours of } j}\left(\operatorname{dis}(t, k, j)-\operatorname{dis}\left(t_{0} k, j\right)\right)^{2}
$$

Where $\operatorname{dis}(t, k, j)$ is the Euclidean distance between points $k$ and $j$ at time $t$. This is the formula for the point $j$ at time $t$. It is an attempt to simulate potential energy of material point subject to Hook law.

To compare time contiguous configuration $C_{t}$ and $C_{t-1}$ we define for each point $j$ and each time $t$

$$
\operatorname{PE} 2(t, j)=\left\|C_{t}-C_{t-1}\right\|
$$

Where $\|$ is the Norm of the vector defined by the point $j$ at time $t$ and $t-1$.

It must be underlined that this artifice has no direct connection with the usual energy definition (this is the reason we use the term pseudo-energy). Actually is only a graphic utility for a better understanding of the deformation. Extended use of them can be find in [10].

\section{Results}

In this section we briefly resume some results obtained by this tool in the preceding works [8], [17] where different strain of the leaders, with different choices, have been investigated. In preceeding papers we also have described the behaviour of some ASTM sample and the respect of Saint Venant principle; but now we want to focus our attention on other simple simulation to outline what we have to improve and what we need to understand better.

So far consider a case of simple strain and release in tensile test of rectangular shape specimen. We are considering a square sample undergoing strain from one side (the other side is clamped) at constant velocity in $\mathrm{x}$ direction (speed 0.6 unit length/step time), with a square lattice 10x10 unit. At a certain time the pull is released and the leaders return to original configuration (this mean that leaders have changed category and are now followers) attracted by the other points. The simple rule, governing follower's motion is that every point must be placed in the barycentre of its neighbours (Eq. 1); the neighbours are determined by the coordination number of the lattice; therefore the leader's motion implies a displacement of the first layer that propagates in successive time steps to the other particles. This means the displacements, at each time step, involve a larger shell of points until to regards all the lattice points. In second gradient [17] we have considered also the neighbours of the first neighbours. In Figure 1 we can see the configuration of the lattice over different time together with the PE1 contour plot. Red points are the leaders; blue the followers and orange the frame. From the figure we can outline that the $\mathrm{x}$ displacement of the points seems do not depend on the $\mathrm{y}$ coordinate; however looking at the PE1 picture we can note a light convexity that does mean this is not true. 

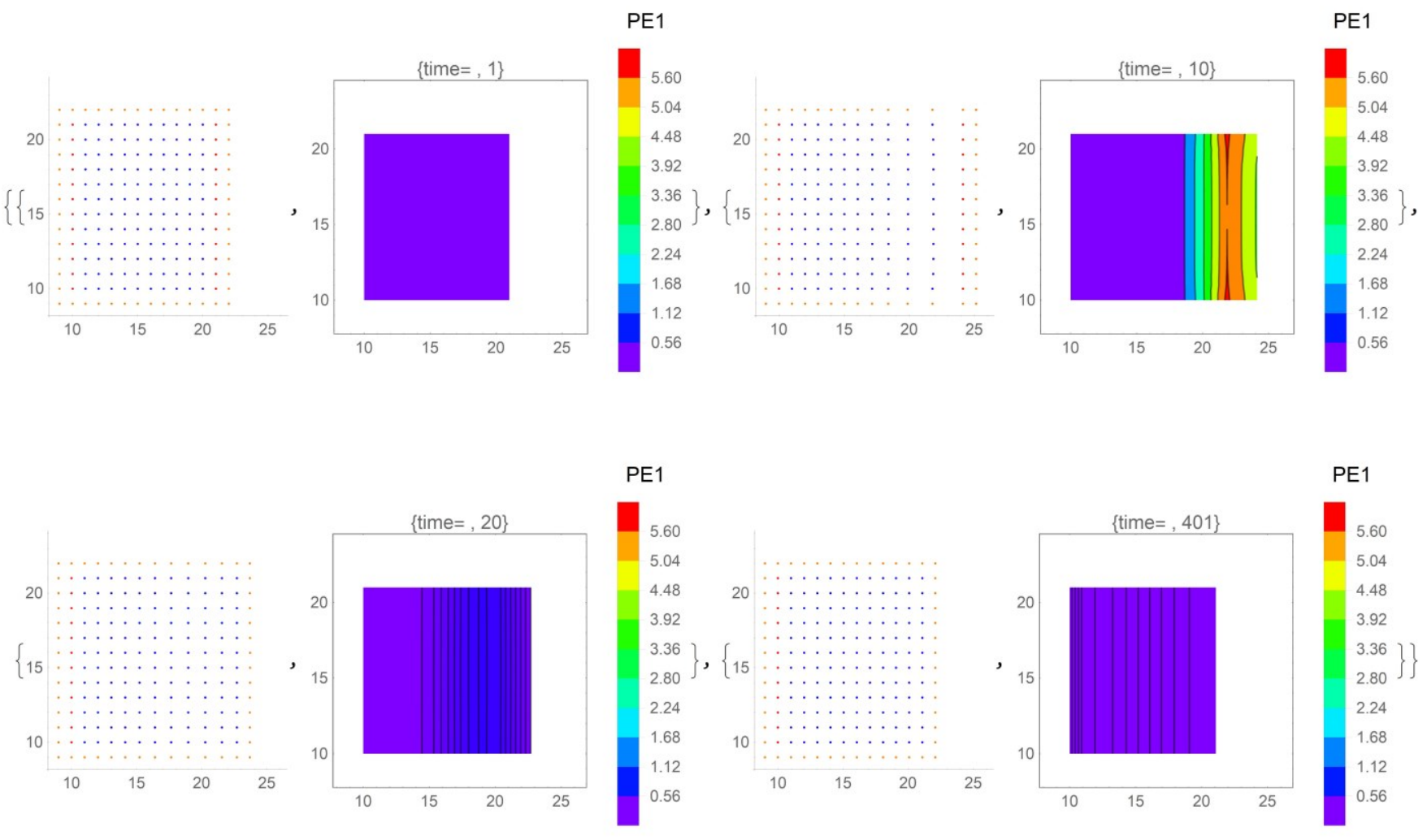

Figure 1 Configuration of the lattice over different time (1,10, 20 and 401) and PE1 contour plot

A deeper examination of the point's displacements confirms as close to the frame the displacements, along $\mathrm{x}$ coordinate, are lower with respect to central points. This can be explained as an edge effect. In fact if we consider points on the same vertical lines those that are close to the frame follow the neighbours with a little delay owing to the different rule determining the displacement of the frame and of the followers. So they see a different situation with respect to, as an example, a central point. Moreover we can note as the maximum value of PE1 (red area) is not on the leader line but just one line on its left; this because, in this case, the leaders have in their neighbours, some points of the frame that always are close to them. We can avoid this convexity effect using a different frame or mirroring the followers to obtain an infinite sample. Finally it should be noted as at $t=401$ the lattice is not back completely to the reference configuration owing to asymptotic process of relaxation. Consider now a central point $j=67$ (sixth column, seventh row, points are numbered from left to right and from bottom to up) of the lattice. The value of the PE1 increases notably when points are pulled, after a delay owing to the propagation time as can be seen in Figure 2; it decrease when the leaders become followers subjected only to the rules leading to equilibrium barycentre position. If we change point the shape of the curve remains the same but can be less o more flared. In Figure 2 and Figure 2 the evolution with time of the coordinates of central point $\mathrm{j}=67$ and close to the leaders $(j=115)$ are shown. Also in this picture we can recognize the coordinate $x$ increases linearly (velocity is constant), after a delay (less for $j=115$ ), owing to the propagation time and later decrease to the original position.

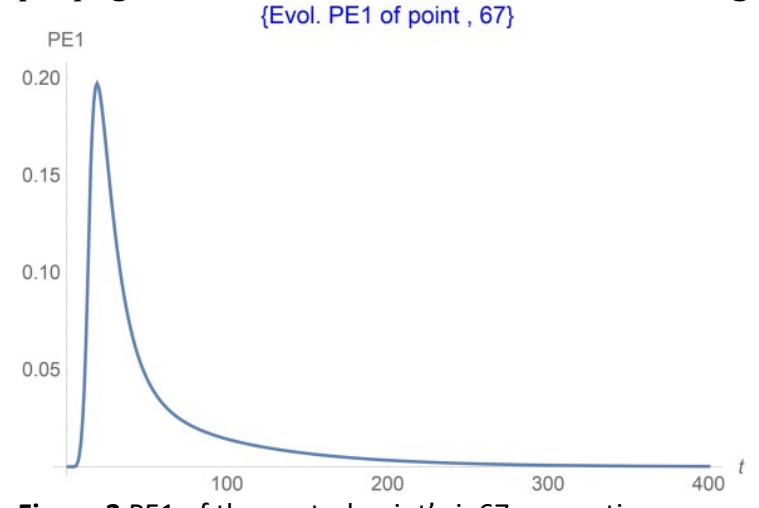

Figure 2 PE1 of the central point's $j=67$ versus time

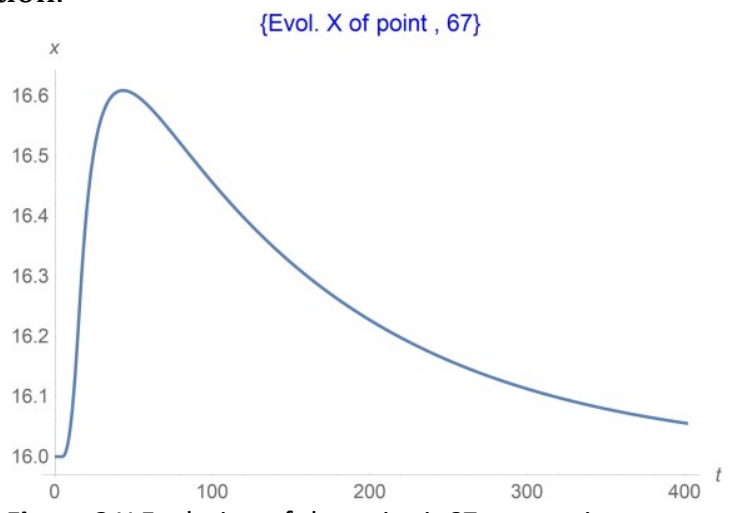

Figure $3 \times$ Evolution of the point $j=67$ versus time 
A light modification can generate instabilities and oscillations; as example we can add to Eq.1 a feedback term proportional to the difference between actual and initial position to overshoot the old equilibrium position. The result is showed in Figure 2; note as also the reaction time is changed.

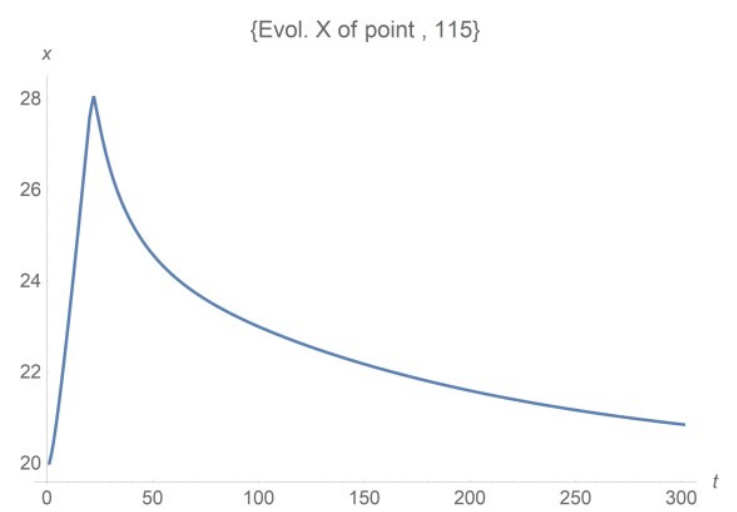

Figure $4 \times$ Evolution of the point $j=115$ versus time

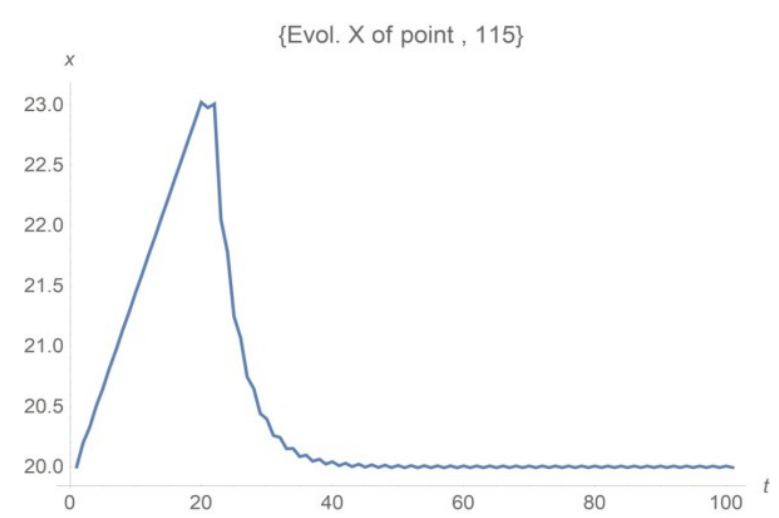

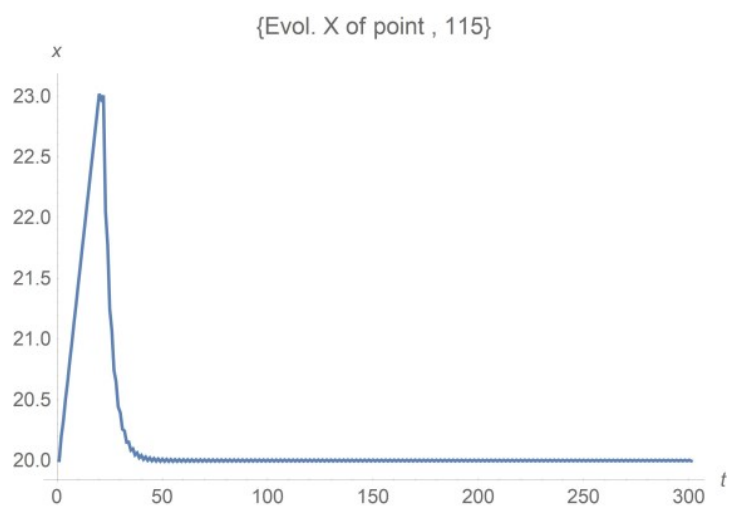

Figure $5 \times$ Evolution $\mathrm{j}=115$ versus time (modified rule with feedback)

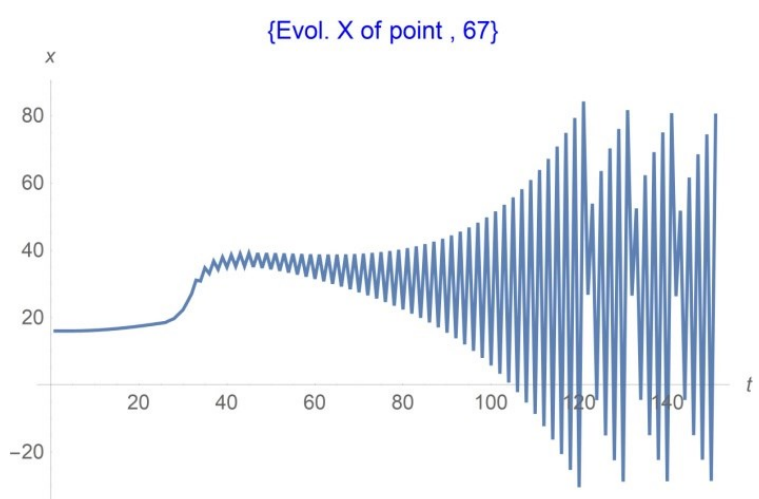

Figure 6 X Evolution $\mathrm{j}=115$ versus time (modified rule with feedback) Figure $7 \times$ Evolution of the point $\mathrm{j}=67$ versus time (modified rule with feedback)

Considering now an ASTM specimen, for tensile test (see Figure8) during fracture. We are using a rectangular centered lattice with neighbor numbers reduced to five, to increase point's mobility. Distance fracture, $d f$, is 11 units and the speed was 0.6step/unit time. The fracture occurs close to the top of the profile and not in the central area. Studies, in progress, show as the fracture zone can be moved by varying working conditions. We can render the fracture more or less brittle changing the model parameters like neighbors' number, type of lattice, speed etc. The PE1 plot in this case is less significant because it does not take in account on the fictitious points; we are working on it to make this parameter meaningful also in fracture case. 


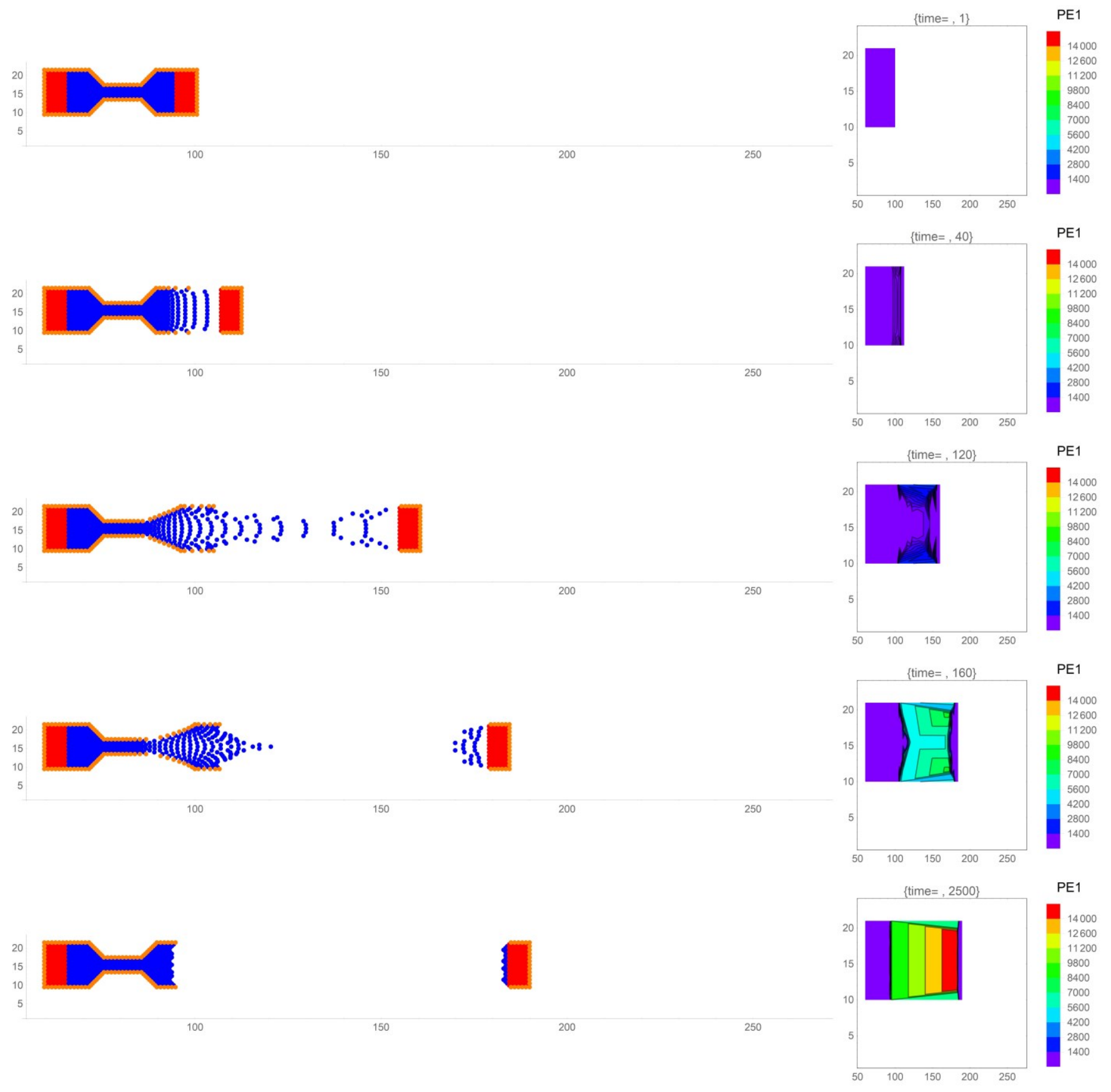

Figure8 ASTM fracture test rectangular centred lattice, coordination number 5.

\section{The beam}

In this section we want to investigate the behaviour of our tool applied to a bending beam loaded at one end and evaluate the differences with the numerical solutions of the differential equations. In the preceding papers we got some success but we need to go deeply to a better understanding so we are looking for some cases that do not fit on what we are expecting. We consider a bi-dimensional elastic short square beam (X and $\mathrm{Y}$ coordinate from 10 to 21 at the not deformed configuration) with materials parameters $Y=1000 \mathrm{~N} /\left(\mathrm{m}^{2}\right)$ and $v=0.33$, where $\mathrm{Y}$ is the Young modulus, $v$ the Poisson coefficient; this value are close to that of a medium molecular weight polypropylene [18]-[20]. Boundary load is on the right (Neumann condition) and no displacements on the left (Dirichelet conditions).

The equations to be solved are: 


$$
\begin{aligned}
& \frac{\mathrm{Y}}{2(1+v)} \nabla^{2} \mathbf{u}+\frac{\mathrm{Y}}{2(1-v)}\left(\frac{\partial}{\partial x} \mathbf{u}+\frac{\partial}{\partial y} \mathbf{v}\right)=0 \\
& \frac{\mathrm{Y}}{2(1+v)} \nabla^{2} \mathbf{v}+\frac{\mathrm{Y}}{2(1-v)}\left(\frac{\partial}{\partial x} \mathbf{u}+\frac{\partial}{\partial y} \mathbf{v}\right)=0
\end{aligned}
$$

$\mathbf{u}(\mathrm{x}, \mathrm{y})$ and $\mathrm{v}(\mathrm{x}, \mathrm{y})$ are the displacements function. We pose as boundary conditions 50 Pascal as shear stress on the beam (Neumann condition for $\mathrm{x}=21$ ) and $\mathbf{u}(10, \mathrm{y})=\mathbf{v}(10, \mathrm{y})=0$ as Dirichelet condition. These equations can be solved numerically; if we discretize our beam by a 10 x 10 square lattice; the solution is shown in Figure 9 and the Von Mises plot in Figure 10; deformed mesh are plotted in red colour.

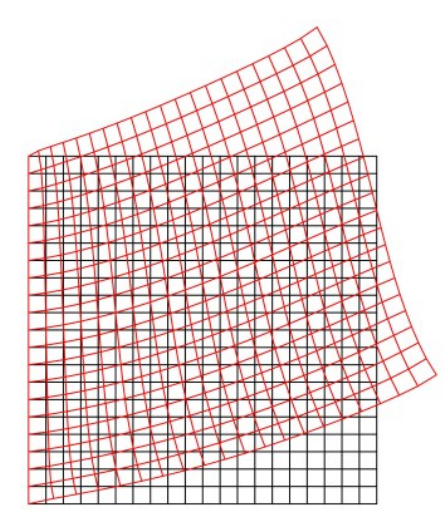

Figure 9 FEM solutions of bidimensional square beam under shear stress

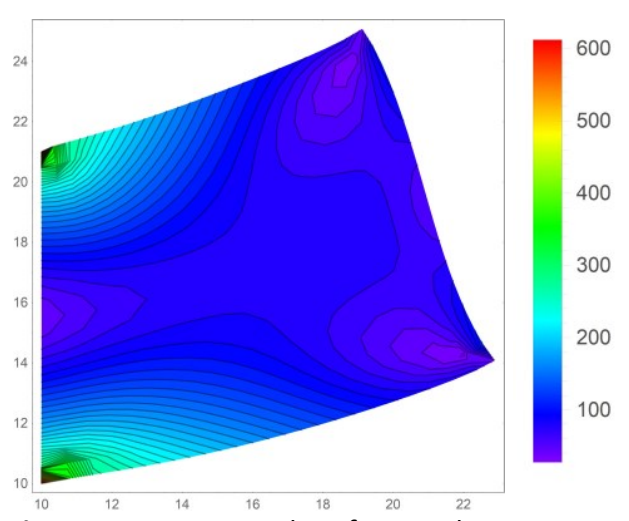

Figure 10 Von Mises plot of FEM solutions

To apply our tool we assign the displacements of some points, the leaders, make some choice about the algorithm (Lattice, interaction rules between the followers etc..) and compute the strain when the followers readjust themselves, after sufficiently long time. As leaders we have chosen the right and left side of the beam, so we assign the displacements of these points as obtained from the FEM equations and investigate the arrangement of the other points. The important thing to point out is that we still have no criteria about the choice of what lattice, interaction law between followers etc... So as first attempt we use a square lattice and no weight in the compute of the followers coordinates In Figure 8 the obtained configuration, together with the FEM solution (red points) are shown; in

Figure 12 the corresponding Von Mises plot. The points on the left and on the right of the beam are overlapped because are the leaders and we have imposed their displacement as the FEM solution beam deformation. It can be outlined as external configuration of the beam is quite the same but the internal displacement of the points, i.e. the strain, is different; this can be highlighted if we look the Von Mises plot. Change in tool's parameters lead to different configuration, corresponding to different strain of the beam, as shown in a paper in press; almost none of them is satisfactory.

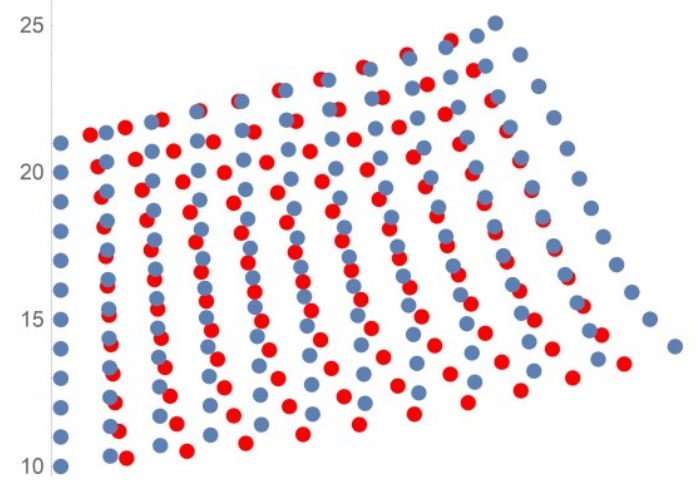

Figure 11 Beam deformation

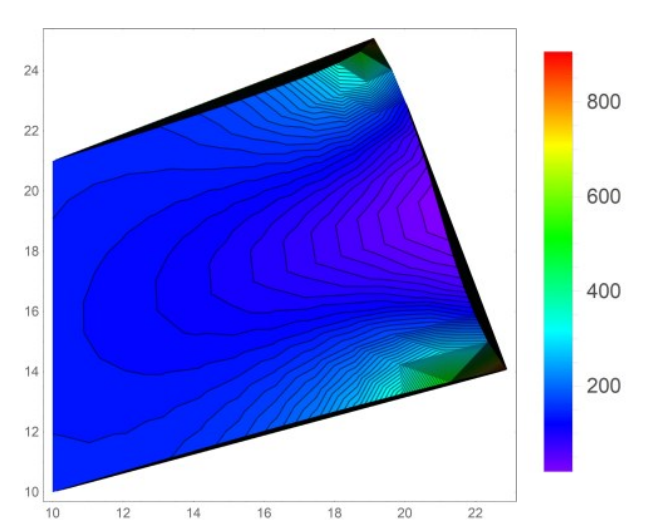

Figure 12 Corresponding Von Mises Plot 

second gradient model; no differences can be appreciated but a quantitative measure of the discrepancies with the FEM solutions, we did in a paper in press, show a light worsening, visible in Von Mises Plot.

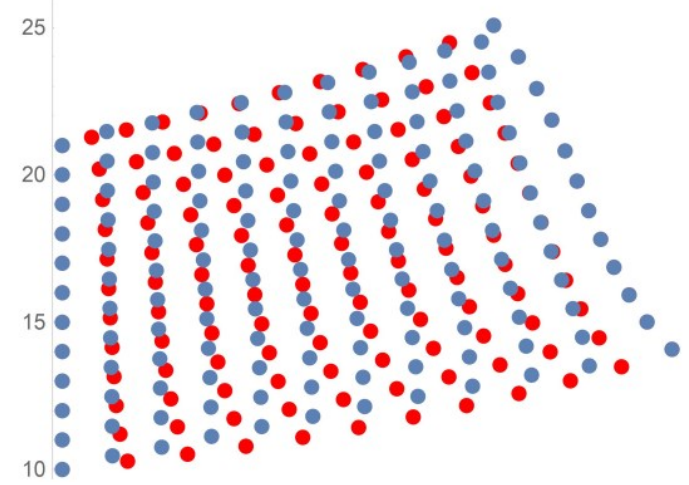

Figure 13 Beam deformation

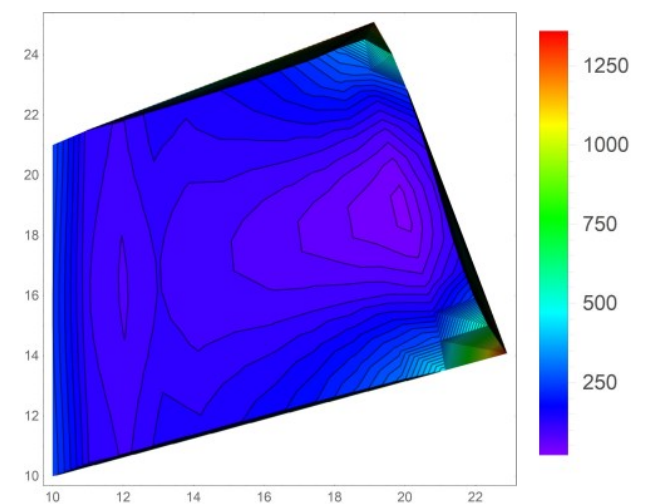

Figure 14 Corresponding Von Mises Plot

We can try many combination of the parameters tool to fit the deflection of our beam but it is meaningless: we need to connect the constitutive parameters with the tool. If we increase the ratio length/width of our beam from 1 to 5 the results are still different (see Figure 12-14). As can be seen the results do not behave as we expected and show a wide range of possibilities in which to choose. Similar results were obtained if we increase the ratio length/width up to 10 or more. Although the external shape of the beam can sometimes be acceptable, we can see that the displacement of the internal particles is quite different from the FEM solutions; it is clear that the differences are sometimes noticeable. This can be evidenced by Von Mises' graphs. In several attempts almost no one emerges as the best. With these results we can conclude that the deformation of the beam can sometimes be very similar to the FEM solution but the stress diagram of Von Mises is quite always unsatisfactory. So far we stop trial and errors to think about the need connection between the tool's parameters and the constitutive features of the material. So far we have to think about the needs to a better understanding of the physic behind the tool and what should be drive our choices in the tool to describe material continuum. We have to remember that the materials parameters $Y$ and $v$ does not appear explicitly in our algorithm but they are hidden into the interaction relationship between the followers, the neighbours and the choice of the lattice. So far we have no idea on how to select our choices to match the problems. This will be the object of the next paper.

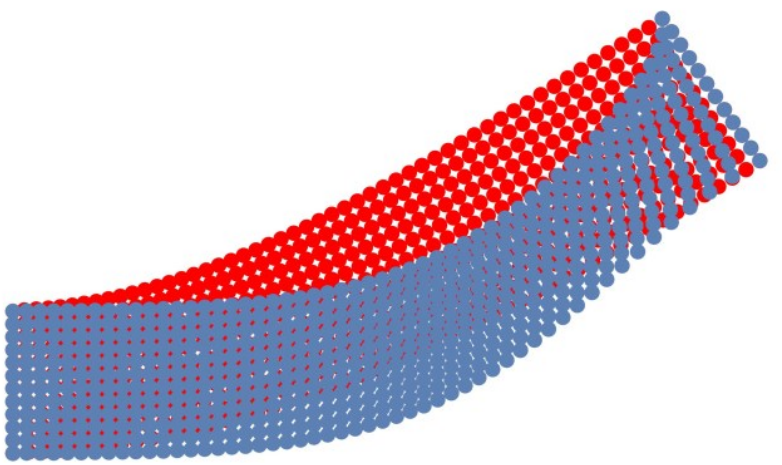

Figure 15 Square lattice

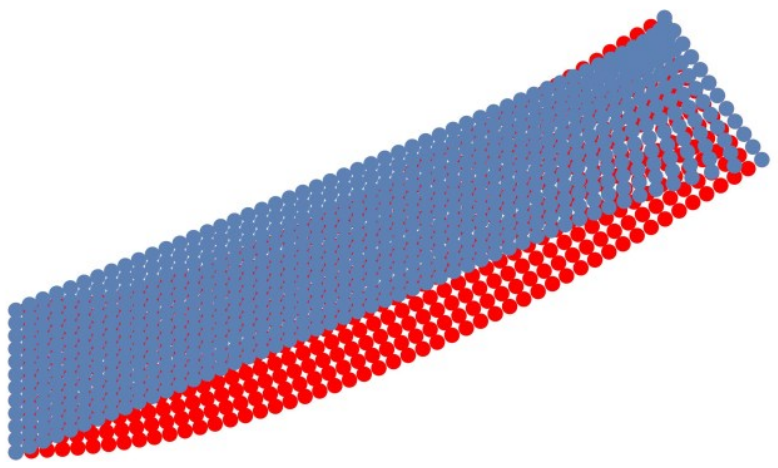

Figure 16 Square lattice reduced neighbours numbers 


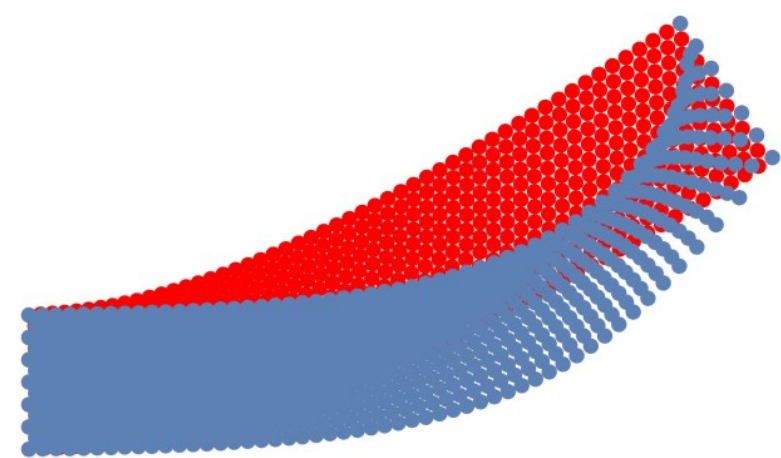

Figura 17 Hexagonal lattice

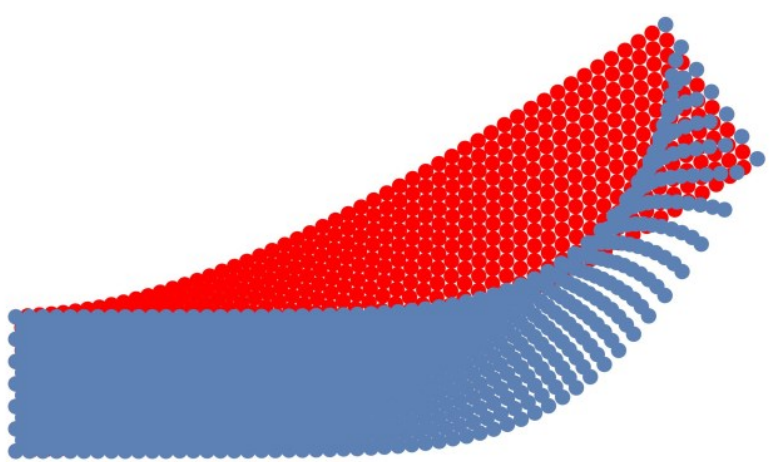

Figure 18 Hexagonal lattice reduced neighbours numbers

\section{Future work and conclusion}

Flocking rule, used in robot swarm, can be used to describe deformation of bi-dimensional continuum medium by a simple algorithm highly customizable and able to adapt to take in account complex physical effects in a plausible way. The strain is imposed on leader's particles whose motion is assigned and the "follower" particles move according to "position rules". These displacements are determined, like in a bird swarm, by the relative position of their neighbours. Therefore the deformed configuration is calculated not by Newton law but only by the relative positions between the particles of the system saving machine time for computing; edge effects are take in account by a frame and fracture mechanism is described by a threshold effect. Changes of some parameters like, lattice, interaction rules, fracture distance, numbers of neighbours lead to different behaviour. In previous works we showed as results of this tool has, in some cases, good similarity with the predictions of standard FEM simulations, also in fracture case. Essentially we have to compute the action of a transformation operator between matrices and the job can be parallelized between the GPU cores of the powerful video card, saving computational cost need to solve FEMs. Pseudo energetic considerations has been introduced to describe different deformation regimes, such as elastic and plastic but in a preliminary form and must to be cleared; the future aim is to potential descriptive interactions depending on the relative distance between the particles, which are able to reproduce the well known physical behaviour. Differently from the PBD methods used in computer graphics, we still do not ask for the knowledge of the velocity and do not introduce some kind of forces to take in account mechanical effects. The presented results are interesting but they still are at a preliminary stage. We have collected some success showing plausible deformation in different conditions but when we consider a beam under loading the need to connect constitutive equations with the parameters of our tool emerges powerfully. So the most important topic is to understand how materials parameters are related with the choices we do in our tool, to make a connection with the usual methods of Continuum Mechanic. Actually we have not criteria about how direct our tool's choices to describe a particular material continuum. In the beam deformation we have used Young modulus and Poisson coefficient assigned but there is no relationship with the parameters of our model. This is the reason of the discrepancy in the resulting deformation. However the tool has demonstrated enough flexibility to give chances that, once connected with the constitutive parameters, we can describe many other behaviour. Generalization in 3D would be easy but needs some optimization in the code to keep the computation time in the order of seconds, by using a normal PC Desktop. We are relaxing the hypothesis of Lagrangian neighbours to describe liquid and gas; this force us to a calculation step more. You have to compute the neighbours, now defined as the particles inside a specified volume, at each time step. Another interesting feature we are introducing is constrained on the particle's motion to describe structured object like pantograph. It can be described as a set of beams with constrained point in the pivot; in the Hencky vision can be conceived as a set of point interconnected by springs. Further developments are concerning different fracture mechanism, different frame to avoid edge effects, other interactions rules and adaptive lattice. Cellular automata seems to be a good candidate to enhance our work; cellular automata is a simple computational mechanism that, for example, changes the colour of each cell on a lattice based on the colour of neighbours cells according to a transformation rule. Some attempts to use them in Mechanics have been done, but one of the principal limits of them is that do not evolve sufficiently, so they quickly reach a limited asymptote in their order of complexity. An evolutionary process involving conflict and competition is needed, like in biology systems. Moreover there is no way to predict the outcome of a cellular process without actually running the process. So even though our decisions are determined, there is no way to predetermine 
what these decisions will be. But the system has succeeded, especially in fluid dynamics to describe complex behaviour. The question posed about if I can work on patterns of information, rather than matter and energy, represent the more fundamental building blocks of reality is still open and we would like to make a connection with our tool.

\section{Funding}

The authors received no financial support for the research, authorship, and/or publication of this article.

\section{References}

[1] J. Bender, M. Müller, e M. Macklin, «Position-Based Simulation Methods in Computer Graphics.», in Eurographics (Tutorials), 2015 [Online]. Available at: https://www.researchgate.net/profile/Jan_Bender/publication/274940214_Position-Based_Simulation_Methods_in_Computer_Gr aphics/links/552cc4a40cf29b22c9c466df/Position-Based-Simulation-Methods-in-Computer-Graphics.pdf. [Consultato: 06-set-2017]

[2] R. dell'Erba, «Determination of Spatial Configuration of an Underwater Swarm with Minimum Data», Int. J. Adv. Robot. Syst., vol. 12, n. 7, pag. 97, lug. 2015.

[3] R. Dell’Erba e C. Moriconi, «Bio-inspired Robotics - it». [Online]. Available at: http://www.enea.it/it/produzione-scientifica/edizioni-enea/2014/bio-inspirede-robotics-proceedings. [Consultato: 15-dic-2014]

[4] R. dell'Erba e C. Moriconi, «High power leds in localization of underwater robotics swarms», IFAC-Pap., vol. 48, n. 10, pagg. 117-122, 2015.

[5] R. Dell'Erba, «The Localization problem for an underwater swarm», Tech. Rep. ENEA, 2012.

[6] C. Moriconi e R. dell'Erba, "The Localization Problem for Harness: A Multipurpose Robotic Swarm», in SENSORCOMM 2012, The Sixth International Conference on Sensor Technologies and Applications, 2012, pagg. 327-333 [Online]. Available at: http://www.thinkmind.org/index.php?view=article\&articleid=sensorcomm_2012_14_20_10138. [Consultato: 04-apr-2014]

[7] R. dell'Erba, "Swarm robotics and complex behaviour of continuum material», Contin. Mech. Thermodyn., mag. 2018 [Online]. Available at: https://doi.org/10.1007/s00161-018-0675-1

[8] R. dell'Erba, "Swarm robotics and complex behaviour of continuum material», Contin. Mech. Thermodyn., pagg. 1-26, 2018.

[9] A. Battista, L. Rosa, R. dell'Erba, e L. Greco, «Numerical investigation of a particle system compared with first and second gradient continua: Deformation and fracture phenomena*», Math. Mech. Solids, pag. 1081286516657889, lug. 2016.

[10] R. dell'Erba, "Position-based dynamic of a particle system: a configurable algorithm to describe complex behaviour of continuum material starting from swarm robotics», Contin. Mech. Thermodyn., vol. 30, n. 5, pagg. 1069-1090, set. 2018.

[11] C. Lanczos, The variational principles of mechanics. Courier Corporation, 2012.

[12] L. Placidi, F. Dell'Isola, N. Ianiro, e G. Sciarra, «Variational formulation of pre-stressed solid-fluid mixture theory, with an application to wave phenomena», Eur. J. Mech. ASolids, vol. 27, n. 4, pagg. 582-606, 2008.

[13] F. Dell'Isola e L. Placidi, «Variational principles are a powerful tool also for formulating field theories», in Variational models and methods in solid and fluid mechanics, Springer, 2011, pagg. 1-15.

[14] F. Dell'Isola, A. Della Corte, L. Greco, e A. Luongo, «Plane bias extension test for a continuum with two inextensible families of fibers: a variational treatment with Lagrange multipliers and a perturbation solution», Int. J. Solids Struct., vol. 81, pagg. 1-12, 2016.

[15] F. Dell'Isola e S. Gavrilyuk, Variational models and methods in solid and fluid mechanics, vol. 535. Springer Science \& Business Media, 2012.

[16] F. dell'Isola, N. Auffray, V. A. Eremeyev, A. Madeo, L. Placidi, e G. Rosi, «Least action principle for second gradient continua and capillary fluids: A Lagrangian approach following Piola's point of view», in The complete works of Gabrio Piola: Volume I, Springer, 2014, pagg. 606-694.

[17] R. dell'Erba, «Position-based dynamic of a particle system: a configurable algorithm to describe complex behaviour of continuum material starting from swarm robotics», Contin. Mech. Thermodyn., pagg. 1-22, 2018.

[18] M. Avella, R. DelL'Erba, e E. Martuscelli, «Fiber reinforced polypropylene: Influence of iPP molecular weight on morphology, crystallization, and thermal and mechanical properties», Polym. Compos., vol. 17, n. 2, pagg. 288-299, 1996.

[19] M. Avella, R. dell'Erba, L. D’Orazio, e E. Martuscelli, «Influence of molecular weight and molecular weight distribution on crystallization and thermal behavior of isotactic polypropylene», Polym. Netw. Blends, vol. 5, n. 1, pagg. 47-54, 1995.

[20] M. Avella, R. dell'Erba, E. Martuscelli, e G. Ragosta, «Influence of molecular mass, thermal treatment and nucleating agent on structure and fracture toughness of isotactic polypropylene», Polymer, vol. 34, n. 14, pagg. 2951-2960, 1993. 